\title{
Post Abortion Care with Misoprostol - A Step towards Reduction of Surgical Interventions
}

\author{
Jahan I ${ }^{1}$, Alam I', Tasnim $\mathrm{T}^{3}$ \\ DOI: https://doi.org/10.3329/jafmc.v17i1.56720
}

\begin{abstract}
Introduction: Treatment with misoprostol as per protocol is becoming widely recognized as a low cost and easy to use means of uterine evacuation. In contrast, post abortion care (PAC) with Dilatation evacuation and curettage (D, E \& C), Manual Vacuum Aspiration (MVA) are costly and complicated procedures specially in inexperienced hands.
\end{abstract}

Objective: To see the outcome of the patients treated with misoprostol as per the guideline.

Materials and Methods: This prospective study was carried out at CMH Momenshahi from October 2016 to March 2017. 50 patients with 1st trimester abortion was selected, treated with directly observed doses of misoprostol. For incomplete abortion $600 \mu \mathrm{gm}$ orally, for missed abortion and blighted ovum $600 \mu \mathrm{gm}$ sublingually, 3 hourly 3 doses. Then followed up after 7 days with an USG of pelvic organs report. Data was collected, processing was done using SPSS version 20 .

Results: Among 50 patients 10 were primigravida, 40 were multigravida. Their age ranged from 20-38 years. 11 had previous miscarriages. Gestational age ranged from 5-12 week. Symptoms were vaginal bleeding in 23, pain abdomen in 15 and both in 12 patients. 28 patients needed parenteral analgesia. Expulsion of product of conception occurred from 30 minutes to 96 hrs after administration of last dose. Surgical evacuation was done in 15 (30\%) patients. Indications were excessive P/V bleeding and retained product of conception. Emergency curettage for bleeding was done in 4 patients. 5 patients needed readmission. All 50 patients attended after 7 days.

Conclusion: Management of 1st trimester abortion with misoprostol as per guideline is highly satisfactory. But it requires patience, effective counselling and follow up. If we can follow these, we can reduce surgical interventions and its complications considerably.

Key-words: Post abortion care (PAC), misoprostol, Blue Top Guidelines of Obstetrical and Gynaecological Society of Bangladesh (OGSB), surgical intervention.

\section{Introduction}

Misoprostol is becoming increasingly recognized as a low cost and easy to use means of uterine evacuation ${ }^{1}$. It is included in the WHO Model List of Essential Medicines for incomplete abortion. It is recommended for use in post abortion care (PAC) by International Federation of Gynecology and Obstetrics (FIGO), American College of Obstetricians and Gynecologists (ACOG) and other international organizations and associations. OGSB had also endorsed the use of misoprostol in post abortion care in Blue Top Guidelines in $2012^{2}$. It has several advantages which include ease of clinical use (the drug can be administered via several routes, can be used in remote areas without need of equipment, can be self-administered, avoids iatrogenic injury and pelvic infection and providers need minimum training), low cost, widely available, relatively stable at room temperature and having few side effects. Studies have reported high satisfaction with its use among women ${ }^{1,3,4,5}$. This method enables women to seek effective care at even primary health care facilities, with non-surgically trained, mid-level providers. It also decreases the burden on tertiary health care facilities; reduce cost to health care systems. It could limit the burden on skilled surgical providers; reduce the need for surgical equipment and supplies, surgical ward, sterilization and anesthesia². It can be administered in OPD basis. As per the Blue Top Guidelines of OGSB the regimen for incomplete abortion is single dose of oral misoprostol: $600 \mathrm{mcg}$ or sublingual misoprostol: $400 \mathrm{mcg}$. For Missed Abortion vaginal misoprostol: $800 \mathrm{mcg}$ or sublingual misoprostol: $600 \mathrm{mcg} 3$ hourly, maximum for 3 doses $^{2}$.

Additional medicines are provided like for pain: Paracetamol 500 $\mathrm{mg}$ or NSAIDs such as ibuprofen $400-600 \mathrm{mg}$ orally three times daily, after meals, antiemetics, antibiotics: Tab. Ciprofloxacin (500 $\mathrm{mg}$ ) two times daily for 7 days and Tab. Metronidazole (400 mg) three times daily for 2 days. A follow-up visit is scheduled after one to two weeks following misoprostol administration to confirm completeness of expulsion. If findings show that abortion is incomplete and the woman is clinically stable, MVA is performed. In the event of infection or clinically significant bleeding, vacuum aspiration should be provided, along with other treatment. The expected effects are cramping, pain abdomen bleeding. Common Side Effects are chills, fever, nausea, vomiting and diarrhoea. Possible complications are heavy bleeding and infection. Warning signs for which women should seek immediate medical care are heavy bleeding, fever that lasts more than a day or starts any day

1. Maj Ishrat Jahan, MBBS, MCPS, DGO, FCPS, Classified Specialist in Obstetrics and Gynaecology, CMH, Dhaka (E- mail: lizarenin@gmail.com 2. Lt Col Md. Iftekharul Alam, MBBS, DLO, MCPS, FCPS, Classified Specialist in ENT, CMH, Dhaka 3. Maj Tafazzula Tasnim, MBBS, DGO, MCPS, FCPS, Classified Specialist in Obstetrics and Gynaecologist, CMH, Dhaka. 
after the misoprostol is taken, severe pain, feeling very sick. Aim of this study is to see the outcome of the patients treated with Misoprostol as per the guidelines, to estimate the complete evacuation of uterus and requirement of surgical evacuation, to see the side effects and complications.

\section{Materials and Methods}

This prospective study was conducted in the Department of Obstetrics and Gynae of $\mathrm{CMH}$, Momenshahi, a 150 bedded military hospital at Mymensingh, Bangladesh over 6 months from October 2016 to March 2017. 50 Patients with incomplete abortion, missed abortion and blighted ovum with uterine size less than 12 weeks were selected by purposive sampling technique. Patients with known allergy to misoprostol, signs of sepsis or active PID, haemodynamic instability, haemorrhagic disorder and current anticoagulation therapy were excluded from study. Patients were treated with directly observed doses of Misoprostol; for incomplete abortion $600 \mu \mathrm{gm}$ orally; for missed abortion and blighted ovum $600 \mu \mathrm{gm}$ sublingually, 3 hourly 3 doses for 30 minutes, then swallowing of the rest of tablet with water. They were given antibiotics and treatment for minor side effect like - NSAID for pain, Paracetamol for fever, ORS for diarrhoea and antiemetics for vomiting. The dose was repeated if the patient vomited within 30 minutes. The drug was given to the patient to take in the morning, so that the emergency (if any) could be met in the daytime. They left hospital if they were stable hemodynamically. Patients were followed up after 7 days with an USG of pelvic organ report.

\section{Results}

In this study out of 50 patients, total 35 patients (70\%) had complete evacuation of uterus and 15 (30\%) patients had incomplete evacuation which needed surgical evacuation (Figure-1). Age group of the patients ranged from 20 to 38 years. Most of the patients belonged to 23-26 years age group (Table-1). $80 \%$ patients were multigravida and $20 \%$ patients were primigravida. Among 50 patients 7 patients had previous 1 miscarriage, 4 patients had previous 2 miscarriages and 39 patients had no miscarriage.

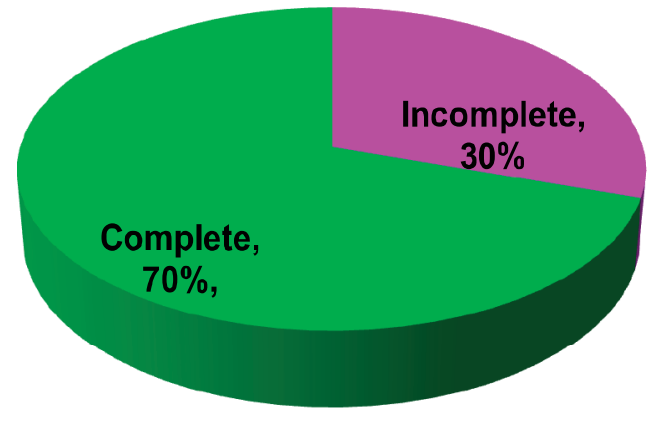

Figure-1: Distribution of patients according to completeness of evacuation of uterus.
Table-I: Distribution of patients according to age.

\begin{tabular}{|l|c|}
\hline \multicolumn{1}{|c|}{ Age Group } & Number of patient \\
\hline $20-22$ & 11 \\
\hline $23-26$ & 18 \\
\hline $27-30$ & 11 \\
\hline $31-33$ & 5 \\
\hline $34-38$ & 5 \\
\hline Grand Total & 50 \\
\hline
\end{tabular}

Among 50 patients period of amenorrhoea ranged from 5 to 20 wks. $62 \%$ patients had $5-10$ weeks of amenorrhoea. $52 \%$ patients presented with vaginal bleeding, $26 \%$ presented with pain abdomen and $22 \%$ presented with both pain abdomen and vaginal bleeding. Among 50 patients 24 patients had incomplete abortion, 18 patients had blighted ovum and 8 patients had missed abortion (Figure-2). 28 patients needed parenteral analgesia and 22 patients didn't need any parenteral analgesia. Only 4 patients developed heavy P/V bleeding for which they underwent emergency curettage. None of the patients developed any infection. So $92 \%$ patients had no complications (Figure-3).

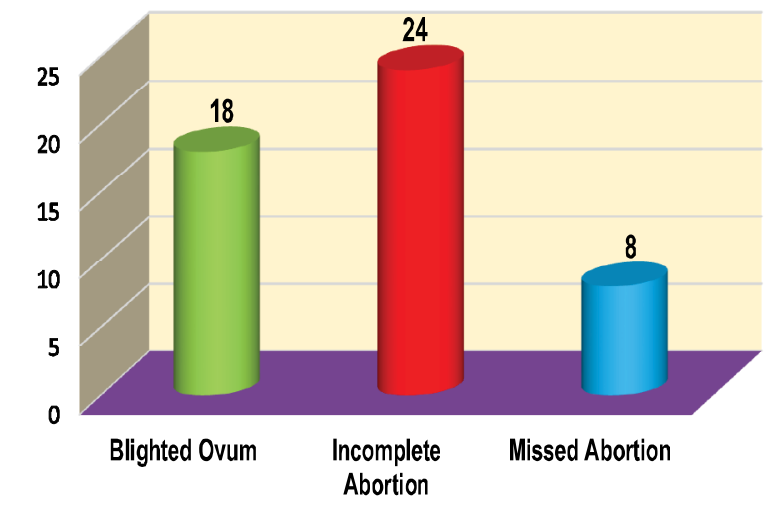

Figure-2: Distribution of patients according to nature of abortion $(n=50)$

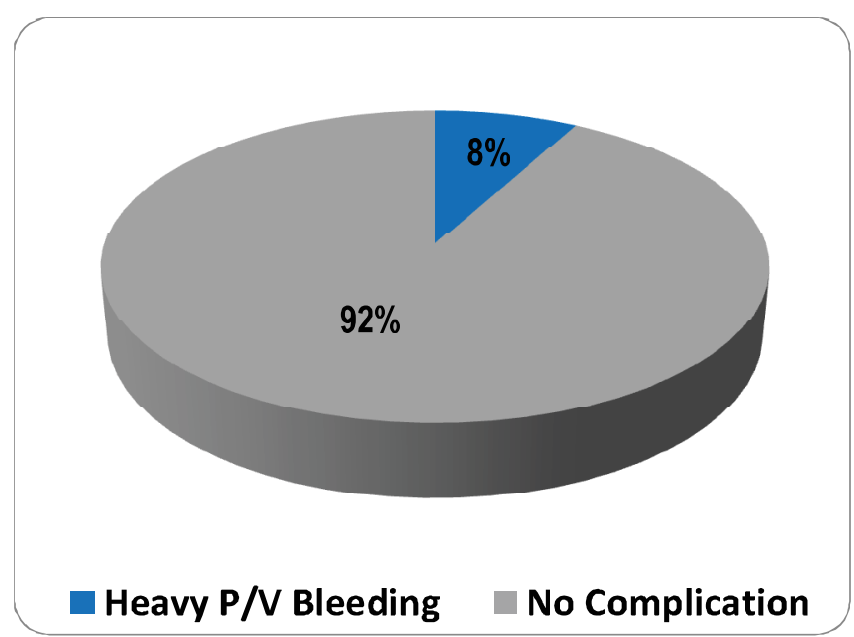

Figure-3: Distribution of patients according to complication $(n=50)$ 


\section{Discussion}

The high proportion of complications related to unsafe abortion continues to pose a major public health challenge in Bangladesh. Our finding shows that treating women with incomplete abortion, missed abortion, ( $\leq 12$ weeks of gestation), with misoprostol was equally effective and safe in a low-resource setting . In addition, the treatment is highly accepted among the treated women.

Misoprostol has been clinically studied in settings as diverse as in US, UK and in low-resource countries such as Burkina Faso, Mozambique and Tanzania for PAC. It has high success rates of about $95 \%$ to $99 \%$, depending on the regimen used3. The overall success rate was $94.8 \%$, which is comparable with the results from other studies that have used the same regimen $(94.5 \%-96.7 \%)^{6-9}$. But in this study success rate was $70 \%$. The result emphasizes that the safety of using misoprostol for treatment of incomplete abortion in low-resource settings, can be performed by trained personnel without the routine use of ultrasonography before or after abortion.

Data from several studies show that as an outpatient care procedure, it substantially reduces the resources used for PAC, cost and average length of women's stay in health facilities. In the present study, only $30 \%$ patients needed surgical evacuation. A study in northern Nigeria showed its high acceptability to women and providers among a largely Muslim population ${ }^{3}$. In this study we have also seen its high acceptability to the patients instead of $D, E$ $\& C$ and MVA. In a study in Nigeria, participating healthcare providers, including doctors, midwives and nurses, reported their satisfaction with and enthusiasm for this method ${ }^{10,11}$. This was same in this study. In different studies more than $90 \%$ of women were satisfied or very satisfied with misoprostol for their treatment ${ }^{12,13}$.

\section{Conclusion}

Misoprostol is becoming increasingly recognized as a low-cost and easy-to-use means of uterine evacuation. It is simple technology that can be used in a range of settings, by a range of providers that can stand alone where MVA is not feasible or be complementary to MVA in existing PAC services. But it requires patience from both provider and patient side.

Treatment of abortion with misoprostol is equally effective, safe and accepted as when administered by physicians in a low-resource setting. Systematically provided contraceptive counselling composed with a wide range of contraceptive choices in PAC is effective to mitigate unmet need for contraception. In areas with shortages and scarce resources doctors would greatly increase women's access to safe and highly accepted PAC. These findings are timely and are needed to accomplish standards and guidelines regarding PAC in Bangladesh.

\section{References}

1. Blum J, Winikoff B, Gemzell-Danielsson K et al. Treatment of incomplete abortion and miscarriage with misoprostol. International Journal of Gynecology and Obstetrics. 2007; 99(1):186-9.

2. OGSB. Standard Clinical Management Protocols and Guidelines. FIGO Initiative for Prevention of Unsafe Abortion Oncology Task-Force. 2012:1-12.

3. Gynuity Health Projects. Guidebook on Misoprostol for Treatment of Incomplete Abortion, 3rd August 2009. Available online at: http://gynuity.org/resources/info/guidebook-on-misoprostol-for-treatment-of-incomplete-abortion/

4. Dao B, Blum J, Thieba B et al. Is misoprostol a safe, effective, acceptable alternative to manual vacuum aspiration for postabortion care? Results from a randomized trial in Burkina Faso, West Africa. British Journal of Obstetrics and Gynecology. 2007; 114:1368-75.

5. Shwekerela B, Kaluuna R, Kipingli R et al. Misoprostol for treatment of incomplete abortion at the regional hospital level: Results from Tanzania. British Journal of Obstetrics \& Gynecology. 2007; 114(11):1363-7.

6. Blandine T, Ouattara AZ, Coral A et al. Sublingual misoprostol as first-line care for incomplete abortion in Burkina Faso. Int J Gynaecol Obstet. 2012; 119:166-9.

7. Renner RM, Brahmi D, Kapp N. Who can provide effective and safe termination of pregnancy care? A systematic review. BJOG. 2013; 120:23-31.

8. Olavarrieta CD, Ganatra B, Sorhaindo A et al. Nurse versus physician-provision of early medical abortion in Mexico: A randomized controlled non-inferiority trial. Bull World Health Organ. 2015; 93:249-58.

9. Klingberg-Allvin $\mathrm{M}$, Cleeve A, Atuhairwe $\mathrm{S}$ et al. Comparison of treatment of incomplete abortion with misoprostol by physicians and midwives at district level in Uganda: A randomised controlled equivalence trial. Lancet. 2015; 385:2392-8.

10. Ipas Nigeria and SOGON. 2011a. Offering misoprostol as an alternative to manual vacuum aspiration for treatment of incomplete abortion in Nigeria: Lessons from a multi-site introduction. Abuja, Nigeria: Ipas.

11. Ipas Nigeria and SOGON. 2011b. Notes from the field: Resource needs and consideration for the introduction of misoprostol into existing PAC services. Abuja, Nigeria: Ipas.

12. Ngoc NT, Blum J, Durocher J et al. A randomized controlled study comparing 600 versus $1200 \mathrm{mcg}$ oral misoprostol for medical management of incomplete abortion, Contraception. 2005; 72(6):438-42.

13. Weeks A, Alia G, Blum J et al. A randomized trial of misoprostol compared with manual vacuum aspiration for incomplete abortion. Obstet Gynecol. 2005; 106(3):540-7. 